\title{
MRI studies of brain size and growth in individuals with congenital heart disease
}

\author{
Alexandra F. Bonthrone, Christopher J. Kelly, Isabel H. X. Ng, Serena J. Counsell \\ Centre for the Developing Brain, School of Biomedical Engineering and Imaging Sciences, King's College London, London, UK \\ Contributions: (I) Conception and design: AF Bonthrone, SJ Counsell; (II) Administrative support: None; (III) Provision of study materials or patients: \\ None; (IV) Collection and assembly of data: AF Bonthrone, CJ Kelly, IHX Ng; (V) Data analysis and interpretation: All authors; (VI) Manuscript \\ writing: All authors; (VII) Final approval of manuscript: All authors. \\ Correspondence to: Prof. Serena J. Counsell. Centre for the Developing Brain, School of Biomedical Engineering and Imaging Sciences, King's College \\ London, London, SE1 7EH, UK. Email: serena.counsell@kcl.ac.uk.
}

\begin{abstract}
Congenital heart disease (CHD) is the most frequent congenital abnormality. Most infants born with CHD now survive. However, survivors of CHD are at increased risk of neurodevelopmental impairment, which may be due to impaired brain development in the fetal and neonatal period. Magnetic resonance imaging (MRI) provides objective measures of brain volume and growth. Here, we review MRI studies assessing brain volume and growth in individuals with CHD from the fetus to adolescence. Smaller brain volumes compared to healthy controls are evident from around 30 weeks gestation in fetuses with CHD and are accompanied by increased extracerebral cerebrospinal fluid. This impaired brain growth persists after birth and throughout childhood to adolescence. Risk factors for impaired brain growth include reduced cerebral oxygen delivery in utero, longer time to surgery and increased hospital stay. There is increasing evidence that smaller total and regional brain volumes in this group are associated with adverse neurodevelopmental outcome. However, to date, few studies have assessed the association between early measures of cerebral volume and neurodevelopmental outcome in later childhood. Large prospective multicentre studies are required to better characterise the relationship between brain volume and growth, clinical risk factors and subsequent cognitive, motor, and behavioural impairments in this at-risk population.
\end{abstract}

Keywords: Congenital heart disease (CHD); brain; magnetic resonance imaging (MRI); brain volume

Submitted Sep 04, 2020. Accepted for publication Oct 21, 2020.

doi: $10.21037 / \mathrm{tp}-20-282$

View this article at: http://dx.doi.org/10.21037/tp-20-282

\section{Introduction}

Congenital heart disease (CHD) affects almost $1 \%$ of UK births, and is the most frequent congenital malformation. Improvements in antenatal diagnosis, cardiac surgery, and perioperative care mean that most infants born with CHD now survive (1). However, children and adults with $\mathrm{CHD}$ who required cardiopulmonary bypass surgery in infancy are at increased risk of adverse neurodevelopmental sequalae (2). The burden of the problem is significant, affecting a wide range of developmental domains, including deficits in neuromotor functions, mild impairments in executive function, attention and memory, difficulties with social interactions and emotional and behavioural maladjustment (3-9), which impact upon future educational achievement, employability and quality of life. Supporting optimal brain development to improve neurodevelopmental outcomes has become a focus of clinical and research efforts in infants born with CHD.

The neurological abnormalities in CHD were initially assumed to be the result of hypoxic ischaemic or embolic brain damage associated with surgery and cardiopulmonary bypass. This was later questioned by the finding that approximately half of newborns with CHD had neurological abnormalities before surgery (10). It is now clear that there is a complex interplay between the circulation and 
brain, involving both fetal and postnatal development. The brain undergoes rapid development during fetal life and in the neonatal period and, during this period of rapid brain development, the brain of fetuses and neonates with critical CHD is at risk due to impaired cerebral oxygen and nutrient delivery. Indeed, brain development in fetuses with CHD slows during the $3^{\text {rd }}$ trimester of pregnancy $(11,12)$ and poor brain growth in fetuses with CHD is associated with reduced cerebral oxygen delivery $\left(\mathrm{CDO}_{2}\right)(13)$.

Head circumference represents a simple and widely available proxy of prenatal brain growth. The American Heart Association acknowledged microcephaly as one of the recognised risk factors for impaired neurodevelopment in children with CHD (2), and has been associated with poorer longer-term outcomes through infancy and school age (14-16). A large nationwide cohort study of Danish singleton infants reported smaller head circumference in many types of CHD including hypoplastic left heart syndrome (HLHS), truncus arteriosus, major ventricular septal defect (VSD), transposition of the great arteries (TGA), Tetralogy of Fallot (TOF) and anomalous pulmonary venous return (17). Only TGA was associated with a smaller head circumference relative to birthweight.

In this review we discuss MRI studies assessing brain volume in individuals with CHD from the fetus to adolescence and describe the relationship between brain volume and subsequent neurodevelopmental outcome. We present the following article in accordance with the Translational Pediatrics reporting checklist.

\section{Fetal brain volume}

Fetal ultrasound provides valuable information regarding brain growth in fetuses with CHD and has demonstrated an impaired trajectory of brain growth from around 28 weeks in a mixed cohort of CHD fetuses (18). However, MRI has a number of advantages over ultrasound including the opportunity to assess the volume of brain tissue types (e.g., white matter, grey matter and cerebrospinal fluid, CSF) and regional volumes in addition to overall brain size.

The first MRI study to identify reduced brain volume in fetuses with CHD was by Limperopoulos and colleagues (11) and this finding has subsequently been replicated in other studies. Over the third trimester, total brain volume and intracranial cavity (ICV) volumes became increasingly smaller in a heterogenous sample of CHD cases, compared to controls, with the highest CSF to ICV ration observed in fetuses with HLHS (11). In a subgroup of this cohort, comprising 18 HLHS fetuses and 30 controls, progressive differences in cortical grey and white matter volumes and subcortical grey matter volumes were observed in the $3^{\text {rd }}$ trimester of pregnancy. There were no differences in lateral ventricle volumes between groups. Of note, in this study, cortical grey and white matter volumes were not significantly reduced in the CHD group prior to 30 weeks gestation. However, impaired cortical folding appeared to precede impairments in regional brain growth and was evident as early as 25 weeks gestation (12). Low cerebroplacental resistance in fetuses with HLHS was significantly associated with decreased cortical grey matter, white matter, subcortical grey matter, and decreased cortical surface area, and the absence of antegrade blood flow in the transverse aortic arch was associated with decreased white matter and subcortical grey matter volumes and a reduced cortical surface area in the right hemisphere (12).

In a cohort of 24 fetuses with TOF, while total brain volume, grey matter volume and subcortical brain volume was reduced, and ventricular volume and external CSF volume was increased, there was no difference in cerebellar volume between fetuses with TOF and controls (19). However, in another study comprising a cohort of 46 fetuses with mixed CHD diagnoses, with the exception of those with septation defects (VSD/ASD), cerebellar volume was significantly decreased, whereas supratentorial volumes were not reduced (20). It is worth noting that this retrospective study compared volumes in CHD cases to normal curves which were derived from fetuses undergoing MRI for suspected fetal abnormalities who were subsequently found to be healthy. Further studies examining fetal cerebellar development across a wide range of CHD diagnoses are required.

\section{The effect of haemodynamic abnormalities on fetal brain development}

Circulatory abnormalities as a result of structural defects in CHD can result in altered cerebral blood flow haemodynamics. The earliest quantitative measurements of the distribution of the fetal circulation was performed by Dawes, who used intravascular flow probes in exteriorised fetal lambs to obtain blood saturation measurements from various chambers and vessels, estimated cardiac output, and blood volumes ejected by both left and right ventricles (21). Subsequent work by Rudolph and Heymann used radionuclide-labelled microspheres to measure organ blood flows, cardiac output, the distribution of blood ejected by the left and right 
ventricles, also in fetal lambs but under more physiological conditions (22). This led to pioneering work describing alterations in the fetal blood circulation in CHD (23), and these animal experiments remain the basis for our current understanding of fetal circulation in CHD today.

The effect of hypoxia on the developing cortex has also been recently investigated using a piglet model (24). Brains from piglets exposed to perinatal chronic hypoxia had a smaller total brain volume, with a significant reduction in cortical grey matter volume and impaired cortical gyrification (24).

Recent advances in fetal MRI allow haemodynamic abnormalities to be assessed in human fetuses in utero. Such approaches have shown that reduced $\mathrm{CDO}_{2}$ and reduced cerebral oxygen consumption in utero are associated with reduced fetal brain size in third trimester fetuses with CHD (13). An increasing number of studies in human infants with CHD have now shown that reduced cerebral oxygen delivery is associated with impaired brain development in the newborn period prior to surgery (25-27). These studies are discussed in more detail below.

\section{Neonatal and infant brain volume}

In line with the smaller brain volumes observed in utero, reduced regional and total brain volumes and biometry measures have been identified in neonates with CHD. However, there is considerable variability in CHD physiologies studied and, in many cases, the sample sizes were relatively small.

MRI based cerebral biometry in 67 infants with CHD prior to surgery showed that infants with CHD had smaller 2D measures of the frontal and parietal lobes, cerebellum and brainstem, and smaller measures of left extra-axial fluid space, but larger CSF spaces in the cranio-caudal interopercular distance compared to controls $(n=36)$. Brainstem area was smaller in infants with single ventricle physiology compared to those with biventricular physiology, but this difference did not remain significant after correction for birthweight, postmenstrual age at scan and sex. Smaller measures in the frontal and parietal lobes was associated with delayed white matter microstructural development on diffusion MRI ( $n=19)$ but white matter injury on MRI was not significantly associated with biometry measures (28). The same group reported significantly smaller measures in frontal and parietal lobes, and brainstem on MRI at 3 months in the CHD sample $(\mathrm{n}=57)$. Significant predictors of biometry measures at this age included weight at 3 months, head circumference at birth, duration of mechanical ventilation, and age at MRI. There was no difference in the rate of brain growth from the neonatal period to 3 months of age between controls and infants with CHD (29).

Using 3D MRI, significantly reduced total and regional brain volumes were observed in a heterogenous sample of 19 infants with CHD [TGA $n=13$, single ventricle physiology $n=4$, interrupted aortic arch $n=1$, cortication of the aorta $(\mathrm{CoA}) \mathrm{n}=1$ ] pre-operatively compared to the same number of controls, after correcting for age at scan and sex. However, in this sample, there was no significant association between total brain volume and type of heart defect, or preoperative clinical factors (30).

While most studies have used image segmentation to delineate specific brain regions, a recent study undertook tensor based morphometry to assess differences in brain volume at a voxel-wise level between a heterogenous sample of 64 infants with CHD (TGA $n=29$, CoA $n=15$, HLHS $n=3$, pulmonary atresia $n=2$, tricuspid atresia $n=2$, TOF $n=7$, pulmonary stenosis $=4$, truncus arteriosus $n=1$ and large VSD $n=1$ ) prior to surgery and 192 age- and sexmatched healthy controls. In contrast to segmentationbased studies which extract a volume for a preselected region (e.g., extracerebral CSF, white matter, hippocampus), tensor-based morphometry analyses examine differences in shape and volume in each voxel across the whole brain independent of global volumetric differences between groups. After accounting for global scaling differences, widespread reductions in tissue volume were observed within the basal ganglia, thalami, corpus callosum, occipital, temporal, parietal and frontal lobes, and right hippocampus and clusters of significant volume expansion in cerebrospinal fluid spaces in infants with CHD, in line with previous segmentation-based approaches (31) (Figure 1).

A number of studies have assessed brain volumes preand post-operatively enabling assessments of brain growth in the peri-operative period. In a sample of 31 infants with TGA, single ventricle physiology and CoA, significantly lower post-operative cortical grey matter volume was observed compared to controls. In addition, gyrification and cortical volumes were reduced in cases with single ventricle physiology compared to those with TGA. Infants requiring balloon arterial septostomy had reduced cortical grey matter volume, and lower birth weight and gestational age was significantly associated with lower pre- and postoperative cortical volumetric parameters (32).

Meuwly and colleagues observed significant reductions 


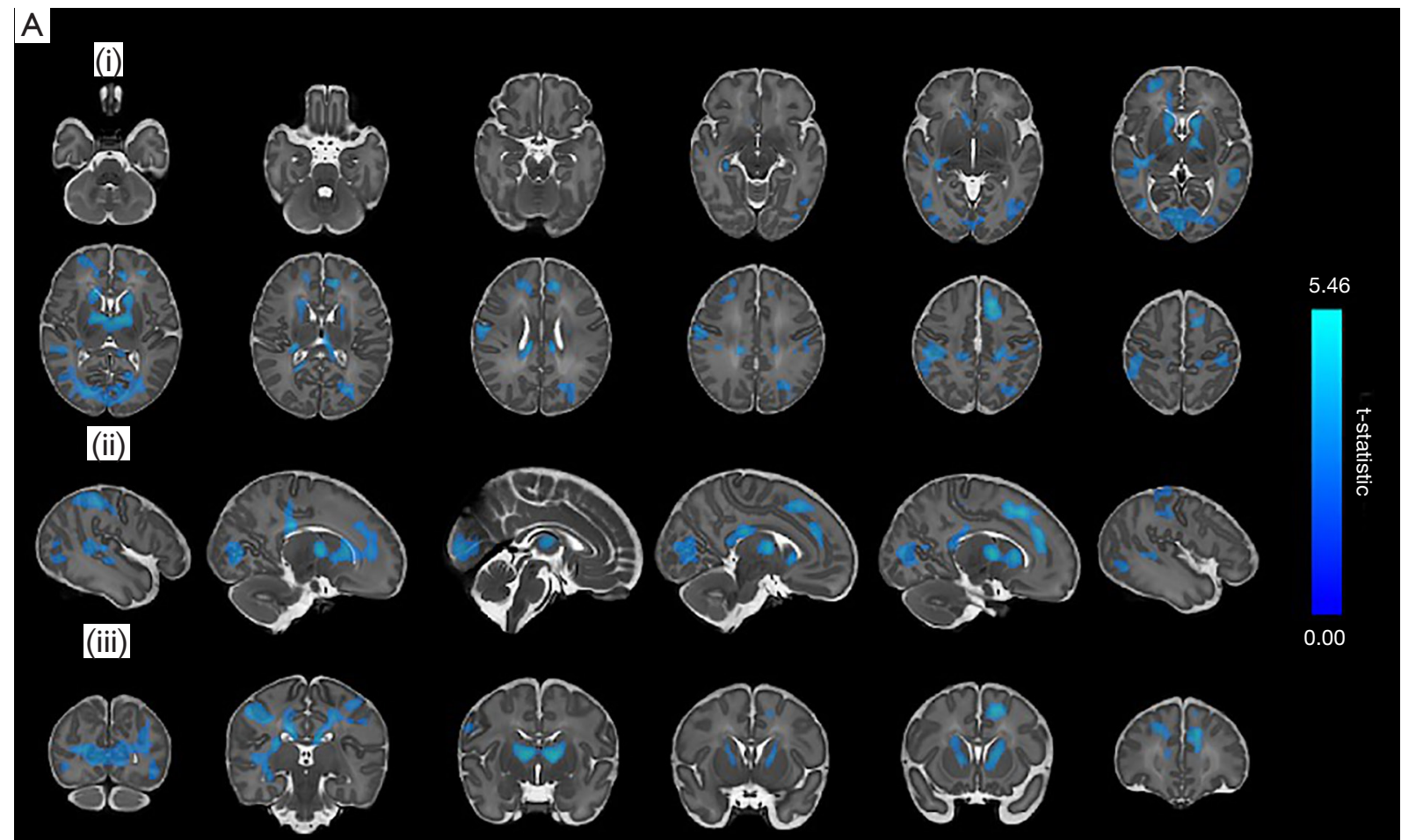

B
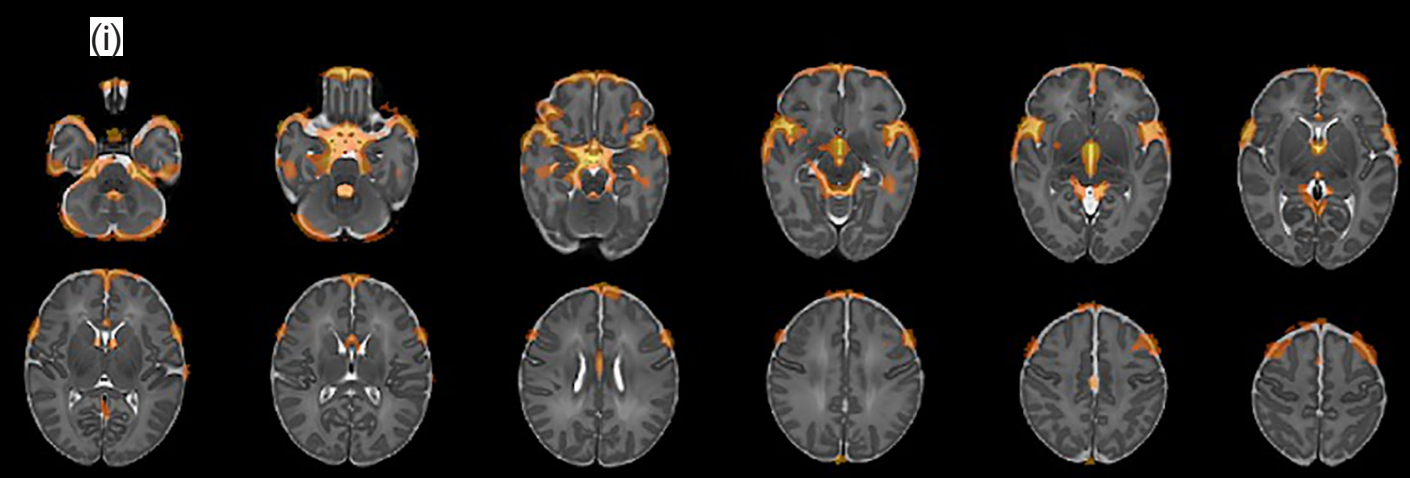

(ii)
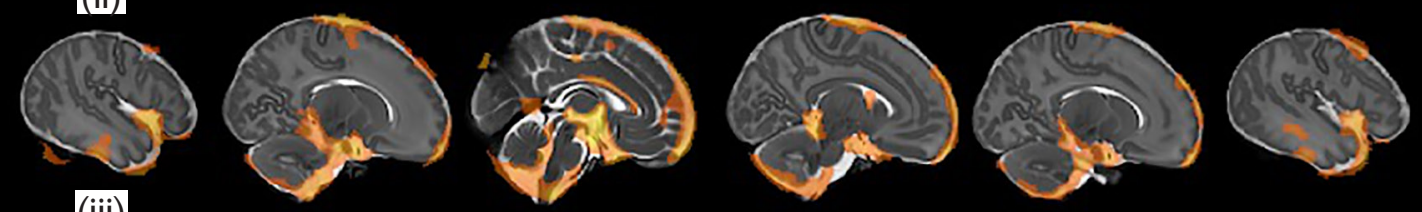

6.90
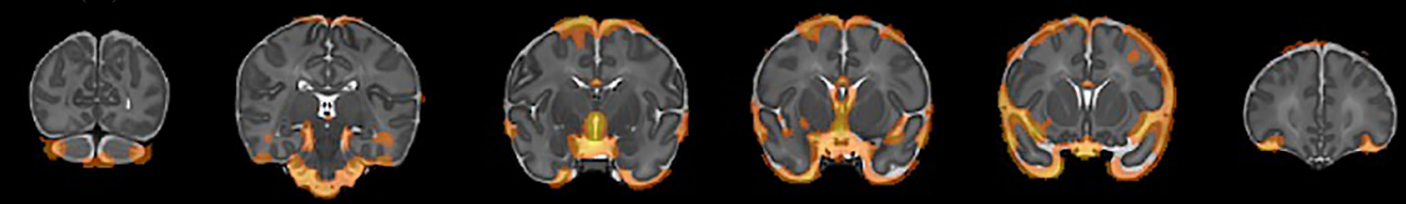

Figure 1 Map of t-statistic values of areas of significant (A) reductions and (B) expansions in volumes in infants with CHD compared to healthy controls (family-wise-error-corrected $\mathrm{P}<0.025$ ). Images in 3 planes are shown: (i) axial, (ii) sagittal, (iii) coronal. t-statistic range is shown on the colour bars. Results are overlaid on the template image with post-menstrual age at scan of 40 weeks. Left-right orientation follows radiological convention. Sagittal views are presented from right to left (31) with permission. CHD, congenital heart disease. 
in pre- and postoperative volumes in CHD infants (67 infants were studied preoperatively and 62 post-operatively) compared to controls $(n=42)$ in total brain, cortical grey matter, white matter, frontal, parietal, occipital, and temporal lobes, and the cerebellum. There were no differences in postoperative whole brain volume between infants with TGA and non-TGA CHD, and no difference between single or biventricular physiologies were identified (33). While preoperative brain volumes were similar, Peyvandi and colleagues demonstrated slower ongoing total and regional brain growth in the perioperative period in HLHS $(n=30)$ compared to TGA $(\mathrm{n}=49)$ infants (34).

Ortinau et al. assessed the brain growth trajectory from the fetus to 3 months after birth and found an altered trajectory of brain growth in the CHD population. Infants with CHD $(n=16)$ had significantly smaller total brain, cortical grey matter, subcortical grey matter, and cerebellar volumes at pre-operative and 3-month MRI compared to controls $(\mathrm{n}=15)$. Infants with CHD also had significantly larger CSF volume at 3-month compared to controls. Brain growth including total brain volume, cortical grey matter, subcortical grey matter, and cerebellar volume was significantly reduced from the fetal period to 3 -month in patients with CHD compared to controls. These differences remained significant when infants with brain injury were excluded. Of note, there was no significant difference in white matter volume or growth between CHD cases and controls (35).

A number of studies report that longer length of hospital stay $(33,36)$ and older age at surgery (37) are significantly associated with reduced total brain volume and growth in this population.

\section{The impact of brain injury on brain volume}

To date, there is little consensus on whether brain volumes are reduced in infants with brain injury on MRI. Claessens et al. investigated 34 infants with aortic arch obstruction and compared brain volumes in infants with and without white matter injury and infarction. They did not identify any differences in postoperative brain volumes or cortical measures between infants with and without moderatesevere white matter injury or focal infarction (38). These findings are supported by other work which found that brain volumes were not significantly different in infants with and without brain injury (39).

Ortinau and colleagues found that the presence of brain injury on MRI was significantly associated with reduced total brain volume at 3 months (35). In infants with TGA $(n=49)$ and HLHS $(n=30)$, mean global, cortical grey matter and deep grey matter volumes were significantly smaller in infants with brain injury compared to those without. However, there was no significant difference in white matter volume between infants with and without brain injury. Pre- and postoperative brain injury were both significantly associated with lower postoperative grey matter volume. Of note though, the strongest predictor of brain growth trajectory was cardiac lesion (see above) and not the presence of brain injury (34).

\section{The relationship between haemodynamic factors and brain development in the neonatal period}

As in the fetal brain, postnatal MRI allows the noninvasive assessment of cerebral oxygenation and most studies that have assessed the relationship between $\mathrm{CDO}_{2}$ and brain volume have observed a significant relationship. $\mathrm{CDO}_{2}$ was significantly reduced in SVP and TGA infants compared to controls, pre-operative brain volume was significantly lower in the CHD sample and there was a significant positive association between $\mathrm{CDO}_{2}$ and brain volumes (25). Similar to these findings, in a heterogenous sample of CHD infants, significant positive correlations were observed between $\mathrm{CDO}_{2}$ and whole brain, cortical grey matter and deep grey matter volumes $(26,27,31)$ along with cortical gyrification (26) and cortical microstructural development (27). However, using tensor-based morphometry, after accounting for global brain size, no significant associations between $\mathrm{CDO}_{2}$ and structural brain development at a voxel-wise level were identified (31). We suggest this reflects that either reduced $\mathrm{CDO}_{2}$ impairs growth across the whole brain with no specific regional vulnerability to low $\mathrm{CDO}_{2}$ in the neonatal period, or that there was insufficient statistical power in our study to determine voxel-wise differences related to $\mathrm{CDO}_{2}$ after correcting for the many multiple comparisons undertaken in voxel-wise analyses.

\section{Genetic influences on brain development in CHD}

The development of the heart and brain are governed by overlapping genetic pathways. Patients with genetic syndromes such as CHARGE and 22q11.2 deletion show cardiac defects, developmental disabilities and reduced brain volumes $(40,41)$. Homsy and colleagues reported that $20 \%$ of patients with CHD and neurodevelopmental 
disabilities showed de novo mutations in genes expressed in the heart and brain (42). In infants with single ventricle physiology $(n=223)$, pathogenic copy number variants were associated with poor outcome scores and impaired growth (43). However, the relationship between genetic variation and volumetric brain development in individuals with CHD has yet to be investigated.

\section{Brain volumes in children, adolescents, and young adults with CHD}

The reduced brain volumes observed in the perinatal period appear to persist into childhood and adolescence. At 1-year, whole brain and frontal lobe volumes were significantly reduced in both TGA $(\mathrm{n}=10)$ and single ventricle physiology $(n=23)$ groups compared with controls $(n=46)$. Temporal lobe volumes were also significantly reduced in the single ventricle physiology group compared to controls. At 3 years, in the same cohort, brain volumes in the infants with TGA were similar to controls but whole brain, frontal lobe and temporal lobe volumes remained significantly smaller in the single ventricle physiology group (44). In an overlapping cohort, total brain volume remained low in children with single ventricle physiology $(\mathrm{n}=18)$ compared to controls at 9 years. Total brain, white matter, basal ganglia, and cerebellum volumes were also significantly smaller in the single ventricle physiology group compared to children with TGA $(n=9)$ at age 9 . Children with single ventricle physiology showed consistently reduced total brain volume from 3 to 9 years compared to TGA indicating a similar rate of brain growth between CHD groups through early and middle childhood. Total brain volume at 9 years was significantly associated with a history of cardiopulmonary resuscitation and time to normalisation of oxygenation levels in children with CHD (45). These findings are supported by a study of 19 patients with HLHS post hybrid procedure, where relative white matter volume was reduced and relative CSF volume increased compared to controls $(n=6)$ at a mean age of 27 months (46).

A large study of 128 children and adolescents (aged 10-19 years) with single ventricle CHD who underwent the Fontan procedure observed reduced total intracranial volume as well as widespread regional volume reductions in temporal and parietal lobes and subcortical grey matter regions including the hippocampus compared to controls $(\mathrm{n}=48)$. Risk factors for reduced brain volumes included increasing numbers of open and catherization procedures as well as higher rates of operative complications. Those adolescents who underwent the Norwood procedure had smaller volumes in several grey matter regions compared to those who underwent other types of surgery (47).

Other studies have examined differences in brain volumes between children and adolescents with cyanotic and acyanotic CHD. In adolescents with CHD who underwent cardiopulmonary bypass surgery $(n=39$; mean age 13.8 years) brain volumes were reduced compared to controls $(\mathrm{n}=32)$ with cyanotic individuals having significantly reduced matter, thalami, hippocampi and corpus callosum volumes compared to those with acyanotic CHD (48). However, in an overlapping cohort of adolescents with CHD ( $n=48$, mean age $=13.8$ years), absolute hippocampal volumes were reduced compared to controls $(n=32)$ but volumes were not significantly different between cyanotic and acyanotic CHD (49).

\section{The relationship between brain volume and neurodevelopmental outcome}

Several studies, spanning the neonatal period to adolescence, have identified associations between brain volumes and neurodevelopmental outcome measures undertaken at around the same time as the neuroimaging examination. For example, difficulty maintaining an alert behavioural state in neonates with CHD $(n=35)$ was associated with increased CSF volume and reduced subcortical grey matter volume on preoperative neonatal MRI and increased CSF volume was also associated with poor visual orienting. In the same study, reduced subcortical grey matter was also associated with significantly higher (representing more abnormal) scores on the Einstein Neonatal Neurobehavioral Assessment Scale in cyanotic infants $(\mathrm{n}=26)$, while in acyanotic infants $(n=9)$, decreased cerebellar volume was associated with poor behavioural state regulation (50).

Multiple studies have examined the relationship between brain volumes and neurodevelopmental outcome in early childhood assessed with the Bayley Scales of Infant Development $2^{\text {nd }}$ Edition (BSID-II) which provides a Psychomotor Development index (PDI) and Mental Development Index (MDI); and the Bayley Scales of Infant and Toddler Development $3{ }^{\text {rd }}$ Edition (Bayley-III) which provides Cognitive, Language and Motor Composite Scores. Frontal and temporal lobe volumes have been linked to early motor and cognitive development in several studies. Increased PDI, but not MDI scores correlated with frontal grey matter volume on post-operative MRI in 40 infants with CHD (mean age 14 months) (51). Another study using 
the BSID-II in 33 infants identified a relationship between larger frontal lobe volumes and higher MDI scores while increased whole brain, frontal and temporal lobe volumes were significantly associated with PDI scores $(n=33)$. Of note, functional oxygen saturation and further surgery in the neonatal period were also associated with PDI scores in this study (44). Poorer cognitive, language and motor composite scores on the Bayley-III have also been linked to increased absolute CSF volumes in toddlers with single ventricle physiology ( $\mathrm{n}=44$; mean age 29 months) (52). Additionally, total brain and regional brain volumes in infants with biventricular CHD $(n=48)$ at one year were significantly associated with language abilities assessed with the MacArthur-Bates Communicative Development Inventories (CDI) but were not correlated with BSIDII scores (53). However, in children with HLHS who underwent the hybrid procedure $(n=19$; mean age 27 months), brain volumes were not associated with BayleyIII scores (46).

At 9 years, full-scale IQ was significantly associated with total brain, total cerebral cortex, temporal cortex, and cerebellar volumes in 27 children with TGA and single ventricle physiology. Temporal cortex volume was also associated with working memory and processing speed indices, while white matter volume was associated with perceptual reasoning index (45).

There is some consensus that impaired hippocampal growth in children with CHD is associated with adverse neurodevelopment. In adolescents with cyanotic and acyanotic CHD at 13.8 years (range, 11-16 years), reduced hippocampal volume was associated with lower total IQ $(48,49)$, perceptual reasoning $(48)$ verbal comprehension and working memory (49). There was no such relationship between hippocampal volume and IQ scores in controls $(n=32)(49)$, however there may be an association between hippocampal volumes and perceptual reasoning in healthy adolescents (48). In children with TGA (age range, 8-16 years), hippocampal volumes, corrected for intracranial volume, were positively associated with general memory, immediate verbal memory, delayed verbal memory and episodic memory scores but, in this study, not full-scale IQ, verbal fluency or academic achievement (54). Interestingly, in this study, children with TGA were not significantly different from controls on measures of IQ, academic achievement or verbal fluency but performed significantly worse on tests of hippocampal function including general, verbal, visual and episodic memory. These findings are supported by work in adolescents and young adults aged
16-24 years $(\mathrm{n}=50)$ where the hippocampi were segmented into subfields: Cornu Ammonis (CA)1, CA2/CA3, CA4/ dentate gyrus (DG) and stratum. Smaller volumes in the left CA1 and left CA2/3 subfields of the hippocampus were significantly associated with worse working memory scores on the Behaviour Rating Inventory of Executive FunctionAdult scale (BRIEF-A) (55).

Additional associations observed between brain volumes and neurodevelopmental outcomes in adolescents with CHD include working memory with total brain and cerebellar volume; verbal comprehension with total brain and white matter volume; perceptual reasoning with total brain volume; static balance with total brain and cerebellar volume and motor outcome with white matter volume (48). These relationships were not present in the control group included in this study however corpus callosum volume was associated with processing speed, and cerebellar volume with fine motor performance (48).

One of the main goals of quantitative neuroimaging in CHD is to identify objective and reproducible imaging biomarkers that are able to predict neurodevelopmental outcome.

One study assessing the relationship between fetal imaging measures and outcome on the Bayley-III at 4-6 months identified a significant association between total brain volume, left and right cingulate fissure depth and frontal Inositol/choline and $\mathrm{N}$-acetylaspartate/choline ratios in the third trimester and average Bayley-III scores (56). However, as the Bayley-III assessment was undertaken in only those cases not requiring open-heart surgery during the first 6 months of age $(n=17)$, larger studies assessing prenatal development and later neurodevelopmental outcome are required.

Postsurgical neonatal brain volumes were associated with Bayley-III scores at 12 months of age in a sample of 62 infants with a range of CHD types. Total brain, cortical, white matter, frontal lobe, temporal lobe, and cerebellar volumes were positively associated with cognitive outcome when adjusted for variables such as sex, postmenstrual age at time of MRI, and SES. In addition, total brain, cortical, frontal lobe, and temporal lobe volumes on postoperative MRI were associated with language performance. However, there were no significant associations between preoperative brain volumes and outcome scores. When results were corrected for multiple comparisons, the relationship between postsurgical brain volumes and outcome scores were no longer significant. Length of ICU stay was negatively associated with cognitive but not with language 
performance. Motor scores were higher in the TGA sample compared to non-TGA patients but there was no significant difference between these groups in cognitive or language scores (33).

Jakab and colleagues assessed the relationship between brain growth, from the pre-operative to post-operative period and neurodevelopment at 12 months in 44 infants with CHD who were operated within the first month of life using deformation-based morphometry. The growth rate of the left posterior perisylvian region around Heschl's gyrus and the anterior planum temporalae was significantly positively correlated with language composite score on the Bayley-III at 12 months. There was no relationship between brain growth and cognitive or motor composite scores (36).

In a longitudinal study assessing children with CHD at 2 and 6 years, lower cognitive composite scores at 2 years and lower full-scale IQ at 6 years was observed in infants who had moderate-severe white matter injury on perioperative MR. While there were no differences in postoperative brain volumes or cortical measures in children with and without moderate-severe white matter injury or grey matter focal infraction, children with full-scale IQ scores below 85 at age $6(n=9)$ had significantly lower postoperative neonatal basal ganglia, thalamus and brainstem volumes compared to children with full-scale IQ at or above $85(\mathrm{n}=21)(38)$.

\section{Conclusions}

In summary, MRI studies have shown that brain volumes are reduced in individuals with CHD from the fetal period to adolescence and young adulthood. It appears that these volume reductions are not specific to particular tissues or regions, rather growth of all brain regions is impaired. These tissue volume reductions are accompanied by increases in extracerebral CSF volume. There is some evidence that brain volumes are most reduced in those with cyanotic CHD, although this finding is not observed in all studies. Reduced cerebral oxygen delivery during fetal life and in the early postnatal period is associated with smaller brain volumes. Other risk factors for impaired brain growth include older age at surgery and perioperative illness severity as indicated by longer times on ITU.

There is increasing evidence that early measures of brain volume and growth are associated with outcome, although large multicentre studies incorporating known clinical risk factors are required to define more precisely the relationship between fetal and neonatal brain volume and subsequent neurodevelopment.

\section{Acknowledgments}

Funding: This research was funded by the Medical Research Council UK (MR/L011530/1), the British Heart Foundation (FS/15/55/31649), and Action Medical Research (GN2630). This work was supported by the Wellcome Engineering and Physical Sciences Research Council Centre for Medical Engineering at Kings College London (WT 203148/Z/16/Z), and by the National Institute for Health Research (NIHR) Biomedical Research Centre based at Guy's and St Thomas' NHS Foundation Trust and Kings College London. I.H.X.N. is supported by the NIHR Academic Clinical Fellowship.

\section{Footnote}

Provenance and Peer Review: This article was commissioned by the Guest Editor (Antonio F. Corno) for the series "Prenatal Diagnosis in Congenital Heart Defects" published in Translational Pediatrics. The article has undergone external peer review.

Conflicts of Interest: The authors have completed the ICMJE uniform disclosure form (available at http://dx.doi. org/10.21037/tp-20-282). The series "Pre-natal Diagnosis in Congenital Heart Defects" was commissioned by the editorial office without any funding or sponsorship. IHXN reports other from National Institute for Health Research, outside the submitted work. SJC reports grants from Medical Research Council (UK), grants from Action for Medical Research, grants from British Heart Foundation, during the conduct of the study. The authors have no other conflicts of interest to declare.

Ethical Statement: The authors are accountable for all aspects of the work in ensuring that questions related to the accuracy or integrity of any part of the work are appropriately investigated and resolved.

Open Access Statement: This is an Open Access article distributed in accordance with the Creative Commons Attribution-NonCommercial-NoDerivs 4.0 International License (CC BY-NC-ND 4.0), which permits the noncommercial replication and distribution of the article with the strict proviso that no changes or edits are made and the original work is properly cited (including links to both the formal publication through the relevant DOI and the license). See: https://creativecommons.org/ 
licenses/by-nc-nd/4.0/.

\section{References}

1. Marelli A, Miller SP, Marino BS, et al. Brain in Congenital Heart Disease Across the Lifespan: The Cumulative Burden of Injury. Circulation 2016;133:1951-62.

2. Marino BS, Lipkin PH, Newburger JW, et al. Neurodevelopmental outcomes in children with congenital heart disease: evaluation and management: a scientific statement from the American Heart Association. Circulation 2012;126:1143-72.

3. Limperopoulos C, Majnemer A, Shevell MI, et al. Predictors of developmental disabilities after open heart surgery in young children with congenital heart defects. J Pediatr 2002;141:51-8.

4. Latal B. Neurodevelopmental Outcomes of the Child with Congenital Heart Disease. Clin Perinatol 2016;43:173-85.

5. Andropoulos DB, Ahmad HB, Haq T, et al. The association between brain injury, perioperative anesthetic exposure, and 12-month neurodevelopmental outcomes after neonatal cardiac surgery: a retrospective cohort study. Paediatr Anaesth 2014;24:266-74.

6. Gunn JK, Beca J, Hunt RW, et al. Perioperative risk factors for impaired neurodevelopment after cardiac surgery in early infancy. Arch Dis Child 2016;101:1010-6.

7. Bellinger DC, Rivkin MJ, DeMaso D, et al. Adolescents with tetralogy of Fallot: neuropsychological assessment and structural brain imaging. Cardiol Young 2015;25:338-47.

8. Calderon J, Jambaque I, Bonnet D, et al. Executive functions development in 5- to 7-year-old children with transposition of the great arteries: a longitudinal study. Dev Neuropsychol 2014;39:365-84.

9. Kasmi L, Bonnet D, Montreuil M, et al. Neuropsychological and Psychiatric Outcomes in DextroTransposition of the Great Arteries across the Lifespan: A State-of-the-Art Review. Front Pediatr 2017;5:59.

10. Limperopoulos C, Majnemer A, Shevell MI, et al. Neurodevelopmental status of newborns and infants with congenital heart defects before and after open heart surgery. J Pediatr 2000;137:638-45.

11. Limperopoulos C, Tworetzky W, McElhinney DB, et al. Brain volume and metabolism in fetuses with congenital heart disease: evaluation with quantitative magnetic resonance imaging and spectroscopy. Circulation 2010;121:26-33.

12. Clouchoux C, du Plessis AJ, Bouyssi-Kobar M, et al.
Delayed cortical development in fetuses with complex congenital heart disease. Cereb Cortex 2013;23:2932-43.

13. Sun L, Macgowan CK, Sled JG, et al. Reduced fetal cerebral oxygen consumption is associated with smaller brain size in fetuses with congenital heart disease. Circulation 2015;131:1313-23.

14. Gaynor JW, Wernovsky G, Jarvik GP, et al. Patient characteristics are important determinants of neurodevelopmental outcome at one year of age after neonatal and infant cardiac surgery. J Thorac Cardiovasc Surg 2007;133:1344-53, 53.e1-3.

15. Limperopoulos C, Majnemer A, Shevell MI, et al. Neurologic status of newborns with congenital heart defects before open heart surgery. Pediatrics 1999;103:402-8.

16. Matos SM, Sarmento S, Moreira S, et al. Impact of fetal development on neurocognitive performance of adolescents with cyanotic and acyanotic congenital heart disease. Congenit Heart Dis 2014;9:373-81.

17. Matthiesen NB, Henriksen TB, Gaynor JW, et al. Congenital Heart Defects and Indices of Fetal Cerebral Growth in a Nationwide Cohort of 924422 Liveborn Infants. Circulation 2016;133:566-75.

18. Zeng S, Zhou QC, Zhou JW, et al. Volume of intracranial structures on three-dimensional ultrasound in fetuses with congenital heart disease. Ultrasound Obstet Gynecol 2015;46:174-81.

19. Schellen C, Ernst S, Gruber GM, et al. Fetal MRI detects early alterations of brain development in Tetralogy of Fallot. Am J Obstet Gynecol 2015;213:392.e1-7.

20. Olshaker H, Ber R, Hoffman D, et al. Volumetric Brain MRI Study in Fetuses with Congenital Heart Disease. AJNR Am J Neuroradiol 2018;39:1164-9.

21. Dawes GS, Mott JC, Widdicombe JG. The foetal circulation in the lamb. J Physiol 1954;126:563-87.

22. Rudolph AM, Heymann MA. The circulation of the fetus in utero. Methods for studying distribution of blood flow, cardiac output and organ blood flow. Circ Res 1967;21:163-84.

23. Heymann MA, Rudolph AM. Effects of congenital heart disease on fetal and neonatal circulations. Prog Cardiovasc Dis 1972;15:115-43.

24. Morton PD, Korotcova L, Lewis BK, et al. Abnormal neurogenesis and cortical growth in congenital heart disease. Sci Transl Med 2017;9:eaah7029.

25. Lim JM, Kingdom T, Saini B, et al. Cerebral oxygen delivery is reduced in newborns with congenital heart disease. J Thorac Cardiovasc Surg 2016;152:1095-103. 
26. Kelly CJ, Makropoulos A, Cordero-Grande L, et al. Impaired development of the cerebral cortex in infants with congenital heart disease is correlated to reduced cerebral oxygen delivery. Sci Rep 2017;7:15088.

27. Kelly CJ, Christiaens D, Batalle D, et al. Abnormal Microstructural Development of the Cerebral Cortex in Neonates With Congenital Heart Disease Is Associated With Impaired Cerebral Oxygen Delivery. J Am Heart Assoc 2019;8:e009893.

28. Ortinau C, Beca J, Lambeth J, et al. Regional alterations in cerebral growth exist preoperatively in infants with congenital heart disease. J Thorac Cardiovasc Surg 2012;143:1264-70.

29. Ortinau C, Inder T, Lambeth J, et al. Congenital heart disease affects cerebral size but not brain growth. Pediatr Cardiol 2012;33:1138-46.

30. von Rhein M, Buchmann A, Hagmann C, et al. Severe Congenital Heart Defects Are Associated with Global Reduction of Neonatal Brain Volumes. J Pediatr 2015;167:1259-63 e1.

31. Ng IHX, Bonthrone AF, Kelly CJ, et al. Investigating altered brain development in infants with congenital heart disease using tensor-based morphometry. Sci Rep 2020;10:14909.

32. Claessens NH, Moeskops P, Buchmann A, et al. Delayed cortical gray matter development in neonates with severe congenital heart disease. Pediatr Res 2016;80:668-74.

33. Meuwly E, Feldmann M, Knirsch W, et al. Postoperative brain volumes are associated with one-year neurodevelopmental outcome in children with severe congenital heart disease. Sci Rep 2019;9:10885.

34. Peyvandi S, Kim H, Lau J, et al. The association between cardiac physiology, acquired brain injury, and postnatal brain growth in critical congenital heart disease. J Thorac Cardiovasc Surg 2018;155:291-300.e3.

35. Ortinau CM, Mangin-Heimos K, Moen J, et al. Prenatal to postnatal trajectory of brain growth in complex congenital heart disease. Neuroimage Clin 2018;20:913-22.

36. Jakab A, Meuwly E, Feldmann M, et al. Left temporal plane growth predicts language development in newborns with congenital heart disease. Brain 2019;142:1270-81.

37. Lim JM, Porayette P, Marini D, et al. Associations Between Age at Arterial Switch Operation, Brain Growth, and Development in Infants With Transposition of the Great Arteries. Circulation 2019;139:2728-38.

38. Claessens NHP, Algra SO, Ouwehand TL, et al.

Perioperative neonatal brain injury is associated with worse school-age neurodevelopment in children with critical congenital heart disease. Dev Med Child Neurol 2018;60:1052-8.

39. Mulkey SB, Swearingen CJ, Melguizo MS, et al. Multitiered analysis of brain injury in neonates with congenital heart disease. Pediatr Cardiol 2013;34:1772-84.

40. Rogdaki M, Gudbrandsen M, McCutcheon RA, et al. Magnitude and heterogeneity of brain structural abnormalities in 22q11.2 deletion syndrome: a metaanalysis. Mol Psychiatry 2020;25:1704-17.

41. Shiohama T, McDavid J, Levman J, et al. Quantitative brain morphological analysis in CHARGE syndrome. Neuroimage Clin 2019;23:101866.

42. Homsy J, Zaidi S, Shen Y, et al. De novo mutations in congenital heart disease with neurodevelopmental and other congenital anomalies. Science 2015;350:1262-6.

43. Carey AS, Liang L, Edwards J, et al. Effect of copy number variants on outcomes for infants with single ventricle heart defects. Circ Cardiovasc Genet 2013;6:444-51.

44. Ibuki K, Watanabe K, Yoshimura N, et al. The improvement of hypoxia correlates with neuroanatomic and developmental outcomes: comparison of midterm outcomes in infants with transposition of the great arteries or single-ventricle physiology. J Thorac Cardiovasc Surg 2012;143:1077-85.

45. Hiraiwa A, Kawasaki Y, Ibuki K, et al. Brain Development of Children With Single Ventricle Physiology or Transposition of the Great Arteries: A Longitudinal Observation Study. Semin Thorac Cardiovasc Surg 2020;32:936-44.

46. Reich B, Heye KN, Wetterling K, et al.

Neurodevelopmental outcome in hypoplastic left heart syndrome after hybrid procedure. Transl Pediatr 2019;8:94-106.

47. Watson CG, Stopp C, Wypij D, et al. Reduced cortical volume and thickness and their relationship to medical and operative features in post-Fontan children and adolescents. Pediatr Res 2017;81:881-90.

48. von Rhein M, Buchmann A, Hagmann C, et al. Brain volumes predict neurodevelopment in adolescents after surgery for congenital heart disease. Brain 2014;137:268-76.

49. Latal B, Patel P, Liamlahi R, et al. Hippocampal volume reduction is associated with intellectual functions in adolescents with congenital heart disease. Pediatr Res 2016;80:531-7.

50. Owen M, Shevell M, Donofrio M, et al. Brain volume and neurobehavior in newborns with complex congenital heart defects. J Pediatr 2014;164:1121-7.e1.

51. Watanabe K, Matsui M, Matsuzawa J, et al. Impaired 
neuroanatomic development in infants with congenital heart disease. J Thorac Cardiovasc Surg 2009;137:146-53.

52. Heye KN, Knirsch W, Latal B, et al. Reduction of brain volumes after neonatal cardiopulmonary bypass surgery in single-ventricle congenital heart disease before Fontan completion. Pediatr Res 2018;83:63-70.

53. Rollins CK, Asaro LA, Akhondi-Asl A, et al. White Matter Volume Predicts Language Development in Congenital Heart Disease. J Pediatr 2017;181:42-8.e2.

54. Munoz-Lopez M, Hoskote A, Chadwick MJ, et al. Hippocampal damage and memory impairment in

Cite this article as: Bonthrone AF, Kelly CJ, Ng IHX, Counsell SJ. MRI studies of brain size and growth in individuals with congenital heart disease. Transl Pediatr 2021;10(8):21712181. doi: 10.21037/tp-20-282 congenital cyanotic heart disease. Hippocampus 2017;27:417-24.

55. Fontes K, Rohlicek CV, Saint-Martin C, et al. Hippocampal alterations and functional correlates in adolescents and young adults with congenital heart disease. Hum Brain Mapp 2019;40:3548-60.

56. Masoller N, Sanz-Corte SM, Crispi F, et al. Midgestation brain Doppler and head biometry in fetuses with congenital heart disease predict abnormal brain development at birth. Ultrasound Obstet Gynecol 2016;47:65-73. 